\title{
Radiation from accelerated particles in shocks
}

\author{
K.-I. Nishikawa ${ }^{1}$, B. Zhang ${ }^{2}$, E. J. Choi ${ }^{3}$, K. W. Min ${ }^{3}$, J. Niemiec ${ }^{4}$, \\ M. Medvedev ${ }^{5}$, P. Hardee ${ }^{6}$, Y. Mizuno ${ }^{7}$, A. Nordlund ${ }^{8}$, \\ J. Frederiksen ${ }^{8}$, H. Sol ${ }^{9}$, M. Pohl ${ }^{10}$, \\ D. H. Hartmann ${ }^{11}$, and G.J. Fishman ${ }^{12}$
}

${ }^{1}$ Center for Space Plasma and Aeronomic Research, University of Alabama in Huntsville, 320 Sparkman Drive, Huntsville, AL 35805, USA email: ken-ichi.nishikawa-1@nasa.gov

${ }^{2}$ Department of Physics and Astronomy, University of Nevada, Las Vegas, NV 89154, USA

${ }^{3}$ Korea Advanced Institute of Science and Technology, Daejeon 305-701, South Korea

${ }^{4}$ Institute of Nuclear Physics PAN, ul. Radzikowskiego 152, 31-342 Krakow, Poland

${ }^{5}$ Department of Physics and Astronomy, University of Kansas, KS 66045, USA

${ }^{6}$ Department of Physics and Astronomy, The University of Alabama, Tuscaloosa, AL 35487, USA

${ }^{7}$ Institute of Astronomy, National Tsing-Hua University , No. 101, Sec. 2, Kuang-Fu Road., Hsinchu, Taiwan 30013, R.O.C.

${ }^{8}$ Niels Bohr Institute, Juliane Maries Vej 30, 2100 Kbenhavn, Denmark

${ }^{9}$ LUTH, Observatore de Paris-Meudon, 5 place Jules Jansen, 92195 Meudon Cedex, France

${ }^{10}$ Institut fuer Physik und Astronomie, Universitaet Potsdam, 14476 Potsdam-Golm, Germany

${ }^{11}$ Department of Physics and Astronomy, Clemson University, Clemson, SC 29634, USA

${ }^{12}$ NASA/MSFC, 320 Sparkman Drive, Huntsville, AL 35805, USA

\begin{abstract}
Recent PIC simulations of relativistic electron-positron (electron-ion) jets injected into a stationary medium show that particle acceleration occurs in the shocked regions. Simulations show that the Weibel instability is responsible for generating and amplifying highly nonuniform, small-scale magnetic fields and for particle acceleration. These magnetic fields contribute to the electron's transverse deflection behind the shock. The "jitter" radiation from deflected electrons in turbulent magnetic fields has properties different from synchrotron radiation calculated in a uniform magnetic field. This jitter radiation may be important for understanding the complex time evolution and/or spectral structure of gamma-ray bursts, relativistic jets in general, and supernova remnants. In order to calculate radiation from first principles and go beyond the standard synchrotron model, we have used PIC simulations. We present synthetic spectra to compare with the spectra obtained from Fermi observations.
\end{abstract}

Keywords. Relativistic jets, Weibel instability, magnetic field generation, particle acceleration, radiation.

We have calculated the radiation spectra directly from our simulations by integrating the expression for the retarded power, derived from the Liénard-Wiechert potentials for a large number of representative particles in the PIC representation of the plasma (Nishikawa et al. 2009, 2011). In order to obtain the spectrum of the synchrotron/jitter emission, we consider an ensemble of electrons selected in the region where the Weibel instability has fully grown and where the electrons are accelerated in the self-consistently generated magnetic fields.

Figure 1 shows how our synthetic spectrum matches with spectra obtained from Fermi observations. Figure 1a shows the observed spectra in $\nu F_{\nu}$ as modeled by Abdo et al. (2009) at five different time intervals. 

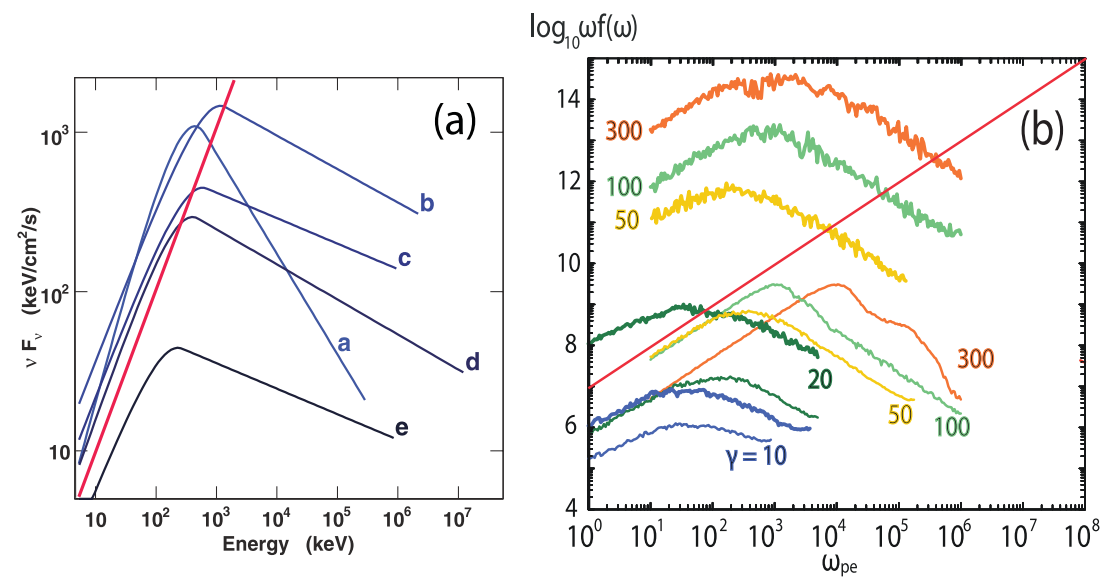

Figure 1. Comparison of a synthetic spectrum with spectra obtained from Fermi observations. Figure 1a shows the modeled Fermi spectra in $\nu F_{\nu}$ units for five time intervals. A flat spectrum would indicate equal energy per decade in photon energy. The changing shapes show the evolution of the spectrum over time. Figure $1 \mathrm{~b}$ shows the spectra for the cases of $\gamma=10,20,50,100$, and 300 with cold (thin lines) and warm (thick lines) electron jets. The low frequency slope is approximately 1.

The red line in Fig. 1a indicates a slope of one, and except for the spectrum at time "a" the low frequency slopes are all approximately one. This is similar to a Bremsstrahlunglike spectrum at least for the low frequency side. As shown in Fig. 1b the slope at low frequency is very similar to the observed spectra. The peaks and slopes at high frequencies change over time.

Emission computed using the method described above is obtained self-consistently, and automatically accounts for magnetic field structures on the small scales responsible for jitter emission. By performing such calculations for simulations using different parameters, we can investigate and compare the different regimes of jitter- and synchrotron-type emission (Medvedev 2006). Thus, we should be able to address the low frequency GRB spectral index violation of the synchrotron spectrum line of death (Medvedev 2006).

\section{Acknowledgements}

This work is supported by NSF-AST-0506719, AST-0506666, AST-0908040, AST0908010, NASA-NNG05GK73G, NNX07AJ88G, NNX08AG83G, NNX08AL39G, and NNX09AD 16G. JN was supported by MNiSW research project N N203 393034 , and The Foundation for Polish Science through the HOMING program, which is supported through the EEA Financial Mechanism. Simulations were performed at the Columbia facility at the NASA Advanced Supercomputing (NAS), and on the IBM p690 (Copper) at the National Center for Supercomputing Applications (NCSA) which is supported by the NSF. Part of this work was done while K.-I. N. was visiting the Niels Bohr Institute. Support from the Danish Natural Science Research Council is gratefully acknowledged. This report was finalized during the program "Particle Acceleration in Astrophysical Plasmas" at the Kavli Institute for Theoretical Physics which is supported by the National Science Foundation under Grant No. PHY05-51164.

\section{References}

Abdo, A. A., et al. 2009, Science, 323, 1688

Medvedev, M. V. 2006, ApJ, 637, 869

Nishikawa, K.-I., et al. 2009b, ApJ, 689, L10

Nishikawa, K.-I., et al. 2011, AdvSR, 47, 1434 